\title{
Enhancing quality in Diffusion Tensor Imaging with anisotropic anomalous diffusion filter
}

\author{
Antonio Carlos da Silva Senra Filho ${ }^{*}$, Carlos Ernesto Garrido Salmon², Antonio Carlos dos Santos ${ }^{3}$, \\ Luiz Otávio Murta Junior ${ }^{1}$
}

${ }^{I}$ Department of Computing and Mathematics, University of São Paulo, Ribeirão Preto, SP, Brazil.

${ }^{2}$ Department of Physics, University of São Paulo, Ribeirão Preto, SP, Brazil.

${ }^{3}$ Department of Medical Clinics, University of São Paulo, Ribeirão Preto, SP, Brazil.

\begin{abstract}
Introduction: Diffusion tensor imaging (DTI) is an important medical imaging modality that has been useful to the study of microstructural changes in neurological diseases. However, the image noise level is a major practical limitation, in which one simple solution could be the average signal from a sequential acquisition. Nevertheless, this approach is time-consuming and is not often applied in the clinical routine. In this study, we aim to evaluate the anisotropic anomalous diffusion (AAD) filter in order to improve the general image quality of DTI. Methods: A group of 20 healthy subjects with DTI data acquired (3T MR scanner) with different numbers of averages ( $\mathrm{N}=1,2,4,6,8$, and 16), where they were submitted to 2-D AAD and conventional anisotropic diffusion filters. The Relative Mean Error (RME), Structural Similarity Index (SSIM), Coefficient of Variation (CV) and tractography reconstruction were evaluated on Fractional Anisotropy (FA) and Apparent Diffusion Coefficient (ADC) maps. Results: The results point to an improvement of up to $30 \%$ of CV, RME, and SSIM for the AAD filter, while up to $14 \%$ was found for the conventional AD filter $(\mathrm{p}<0.05)$. The tractography revealed a better estimative in fiber counting, where the AAD filter resulted in less FA variability. Furthermore, the AAD filter showed a quality improvement similar to a higher average approach, i.e. achieving an image quality equivalent to what was seen in two additional acquisitions. Conclusions: In general, the AAD filter showed robustness in noise attenuation and global image quality improvement even in DTI images with high noise level.
\end{abstract}

Keywords Anomalous diffusion, Image enhancement, Diffusion Tensor Imaging, Spatial filtering.

\section{Introduction}

Diffusion tensor imaging (DTI) is a widely used magnetic resonance imaging (MRI) modality for the study of microstructural changes in neurological diseases and plays an important role in several medical procedures (Alexander et al., 2007; Shenton et al., 2012). However, a major practical limitation of DTI is due to the low image quality, been resultant from imaging acquisition factors such as the spatial resolution and the long echo time necessary to DTI imaging technique. As a consequence,

This is an Open Access article distributed under the terms of the Creative Commons Attribution License, which permits unrestricted use, distribution, and reproduction in any medium, provided the original work is properly cited.

How to cite this article: Senra ACS Fo, Salmon CEG, Santos AC, Murta LO Jr. Enhancing quality in Diffusion Tensor Imaging with anisotropicanomalousdiffusionfilter.ResBiomedEng.2017;33(3):247-258.DOI: $10.1590 / 2446-4740.02017$

*Corresponding author: Department of Computing and Mathematics, University of São Paulo - USP, Av. Bandeirantes, 3900, Monte Alegre, CEP 14040-900, Ribeirão Preto, SP, Brazil. E-mail: acsenrafilho@usp.br

Received: 25 April 2017/ Accepted: 14 August 2017 the accuracy of the quantitative indexes estimated on DTI image reconstructions, such as the fractional anisotropy (FA) and apparent diffusion coefficient (ADC), is compromised. In principle, one simple solution to improve the image quality in DTI exams is to increase the number of images acquired. Thus, the average signal should decrease the noise level on the overall image. However, this approach is time-consuming, being often impractical for the clinical routine.

Another suitable approach to reducing the noise influence is the application of computational noise attenuation algorithms. In fact, several approaches were presented in the literature in order to enhance the general DTI image quality. For instance, some of these DTI filtering methods are based on MRI noise regularization (Aja-Fernandez et al., 2008; Martin-Fernandez et al., 2009; Maximov et al., 2012; Tristán-Vega and Aja-Fernández, 2010), pre-filtering DWI images requested for DTI images reconstruction (Martin-Fernandez et al., 2009; Xu et al., 2010), noise attenuation on k-space (Basu et al., 2006), local neighborhood pattern analysis (Manjón et al., 2008), high-order partial differential equations (Bai and Feng, 2007; Moraschi et al., 2010), diffusion tensor 
modeling (Chen and Hsu, 2005), and image restoration (Christiansen et al., 2007; McGraw et al., 2004).

The anisotropic diffusion AD filter approach was introduced by Perona and Malik (Perona and Malik, 1990) and have been intensively applied on several medical imaging techniques (Lee et al., 2006; Ling and Bovik, 2002; Xu et al., 2010), and recently on DTI images (Moraschi et al., 2010). However, the AD filter applicability is only robust on specific regions of interest, mainly on areas where exist dense fiber concentrations, such as the corpus callosum (Moraschi et al., 2010). This regional limitation also reveals issues on the pericortical brain areas, where the AD filter presents higher structural distortions due to the noise influence. As reported, the AD filter has lower performance on images with both low spatial resolution and high noise level (Manjón et al., 2008).

The main limitation given in the $\mathrm{AD}$ approach is regarding the local voxel interaction, which is restricted to a linear power law described by the classical Brownian motion. In fact, the AD filter is a Gaussian filter locally modulated by tissue boundaries. Perona and Malik (Perona and Malik, 1990) proposed a monotonically decreasing function which, in general terms, regulates the Gaussian variance at each voxel position regarding the local image gradient (Perona and Malik, 1990). However, the fundamental noise smoothing procedure relies on a Gaussian modulation, imposing the classical diffusion paradigm for all brain tissues. Several studies affirm that the brain environment share characteristic of complex systems (Bullmore et al., 2009; Kiselev et al., 2003), arguing that an anomalous diffusion process (ADP) is naturally presented in the neuroanatomical organization.

Inspired by ADP properties, the anisotropic anomalous diffusion (AAD) filter is a spatial image filtering method, which adds the superdiffusion and subdiffusion regimes. Similarly, with the AD approach, the AAD filter modulates the diffusion behavior based on the local diffusibility properties. Although instead of the classical Brownian motion assumption, used in $\mathrm{AD}$ method, the $\mathrm{AAD}$ filter applies the ADP defined by the generalized diffusion equation (also known as the porous media equation), proposed by Tsallis (Senra et al., 2015; Tsallis, 2009). Therefore, instead of assuming a linear diffusion, it is possible to investigate which type of spatial filtering approach is more suitable for diffusion weighted image volumes. More details about the AAD filter and concepts of anomalous diffusion on digital images are discussed in section Anomalous and classic diffusion paradigm.

In this study, the overall image quality enhancement in quantitative maps, FA and ADC, with the proposed AAD filter was analyzed, being helpful to increase the image signal to noise ratio (SNR). In sections Subjects and Anomalous and classic diffusion paradigm, the details about the subjects and the AAD filter paradigm are explained, respectively. The sections Image analysis and quality metrics and Statistical analysis present the quantitative analysis and sections Results and Discussion the study is presented.

\section{Methods}

\section{Subjects}

DTI images from 20 healthy asymptomatic right-handed subjects, with an average age of $28.4 \pm 6.5$ years (13 men, 7 women) were acquired on a 3T MR scanner (Achieva, Philips) at Clinical Hospital of Ribeirão Preto and all the subjects were recruited with a consent form approved by the local ethics committee. The DTI parameters used here was: 2D SE-EPI sequence, $\mathrm{TE}=65 \mathrm{~ms}, \mathrm{TR}=7.4 \mathrm{~s}, \mathrm{~b}$-factor $=1000 \mathrm{~s} / \mathrm{mm}^{2}, 16$ volumes (1 non-diffusion volume and 15 whole-sphere gradients), acquisition matrix $128 \times 128$, voxel resolution $2 \times 2 \times 2 \mathrm{~mm}^{3}, 72$ axial slices for whole brain coverage and sixteen acquisitions. All imaging parameters were set to optimize the total acquisition time in the scanner ( 2 min for $\mathrm{N}=1$ ), based on the clinical application settings. The acquisitions were combined to obtain images with different noise intensity from different numbers of signal averages $(\mathrm{N}=1,2,4,6,8$ and 16). Additionally, $3 \mathrm{D}-\mathrm{T} 1$ weighted images $(3 \mathrm{D}-\mathrm{T} 1 \mathrm{w})$ were also acquired using a gradient echo pulse sequence covering the whole brain with the following parameters: TI/ Flip angle/ TE/ TEchoSpacing/ TR/ Resolution $=900 \mathrm{~ms} / 8 \% 3.2 \mathrm{~ms} /$ $7.0 \mathrm{~ms} / 2500 \mathrm{~ms} / 1 \mathrm{x} 1 \mathrm{x} 1 \mathrm{~mm}^{3}$.

\section{Anomalous and classic diffusion paradigm}

The AAD filter derives from the anomalous diffusion equation and share its features (Tsallis, 2009). The main difference between the AAD filter and the AD filter is the use of q-Gaussian probability distributions (Tsallis, 2009) generated iteratively by the filtering process (Senra et al., 2015). In previous studies, we have shown that q-Gaussian distributions are more suitable for noise attenuation in MRI structural images (T1 and T2 weighted images) than classical Gaussian distribution used in the AD filter (Senra et al., 2013, 2014, 2015). One important feature that arises with the q parameter (also known as the anomalous parameter) is the super and sub diffusivity regimes (Tsallis, 2009), which is related to the generalized nonlinear power law (Schwämmle et al., 2008; Senra et al., 2015). Furthermore, the usual classic diffusion behavior, that is already described by the $A D$ formulation is also presented in AAD filtering process when it is assumed $\mathrm{q}=1$. For $\mathrm{q}<1$, a class of $\mathrm{q}$-Gaussian functions with compact support is defined, which presents the sub-diffusion behavior. On the contrary, for $\mathrm{q}>1$, the q-Gaussian function class with infinite support is obtained, and thereby defines the superdiffusion regime. More details on such probability distributions can be found 
in previous studies (Malacarne et al., 2001; Senra et al., 2015; Tsallis, 2009; Tsallis and Lenzi, 2002).

The time $(\mathrm{t})$, conductance $(\kappa)$ and numerical stability $(\lambda)$ variables were set based on previous studies, selecting its optimum values, i.e., $\mathrm{t}=4 \mathrm{~s}, \kappa=10$ a.u., $\lambda=0.0625$ a.u. (Moraschi et al., 2010; Perona and Malik, 1990; Senra et al., 2015). The Equation 1 defines the anisotropic anomalous diffusion paradigm and Equations 2 and 3 defines the edge modulation function and the generalized diffusion coefficient, respectively (Senra et al., 2015). The $\alpha$ variable is defined as $\alpha=(2-q)(3-q)$, where it is easy to check that when $\mathrm{q}=1$ all equations return the classical anisotropic diffusion method (Perona and Malik, 1990). The iterative numerical procedure applied here is explained in details in (Senra et al., 2015). In addition, the AAD filter is freely available as an open-source code for research applications, where it have been developed as a 3D Slicer module (Senra, 2017) and also as a ITK image processing code (Senra and Murta, 2017).

$$
\begin{aligned}
& \frac{\partial I(\vec{r}, t)}{\partial t}=\lambda \cdot D_{q}(\vec{r}, t) \cdot \nabla^{2} I(\vec{r}, t)^{(2-q)} \\
& D_{q}(\vec{r}, t)=D_{q} \cdot \exp \left(-\frac{|\nabla I(\vec{r}, t)|^{2}}{\kappa^{2}}\right) \\
& D q=\left\{\begin{array}{l}
\frac{1}{2} \cdot \alpha \\
\frac{1}{2} \cdot \alpha^{\frac{2}{3-q}} \cdot\left(\begin{array}{l}
\left.\frac{\sqrt{1-q}}{\pi} \cdot \frac{\Gamma\left(1+\frac{1}{1-q}\right)}{\Gamma\left(\frac{3}{2}+\frac{1}{1-q}\right)}\right)^{\frac{2-2 q}{3-q}}, q<1 \\
\frac{1}{2} \cdot \alpha^{\frac{2}{3-q}} \cdot\left(\frac{\sqrt{q-1}}{\pi} \cdot \frac{\Gamma\left(\frac{1}{q-1}\right)}{\Gamma\left(\frac{1}{q-1}-\frac{1}{2}\right)}\right)^{\frac{2-2 q}{3-q}}, q<q<2
\end{array}\right.
\end{array}\right\}
\end{aligned}
$$

It worth noting that the optimal conductance parameter can be estimated by iterative methods (Tsiotsios and Petrou, 2013), however, we have a fixed $\kappa$ based on the previous optimization in MRI and DTI studies (Moraschi et al., 2010; Senra et al., 2015). Moreover, an optimization strategy was adopted in order to adjust the AAD filter parameters with the same optimum parameters set found for the AD approach, which provides a comparison between the anomalous filter with the best results found with the classical diffusion approach. Furthermore, all filtering processes were performed after the DTI data been pre-processed, i.e. after the eddy current, motion corrections and averaging steps. Therefore, each corrected DTI gradient volumes $(\mathrm{N}=1,2,4,6,8$ and 16) can be filtered with both AAD and $\mathrm{AD}$ spatial filters and then passed to the sequential white matter analysis as presented in Figure 1.

\section{Image analysis and quality metrics}

The FMRIB Software Library (FSL) DTI processing pipeline (Jenkinson et al., 2012) was used to reconstruct the Fractional Anisotropy (FA) and Apparent Diffusion Coefficient (ADC) maps from all the DTI data, following intra-subject affine (12 degrees of freedom) registration for movement and DTI eddy current artifact correction. White matter segmentation was performed using the FSL-FAST tool on individual 3D-T1w images (Figure 1) in which this was applied to the registered DTI quantitative maps in order to obtain the segmented WM on FA and ADC maps.

The image quality resulting from the $\mathrm{AAD}, \mathrm{AD}$ and $\mathrm{N}$ averaging approaches was evaluated on two different image features: quantitative improvement and perceptual image quality. The perceptual image quality was quantified using the structural similarity index (SSIM) (Wang et al., 2004) due to several applications on this specific visual assessment (Lin and Jay Kuo, 2011). For the quantitative

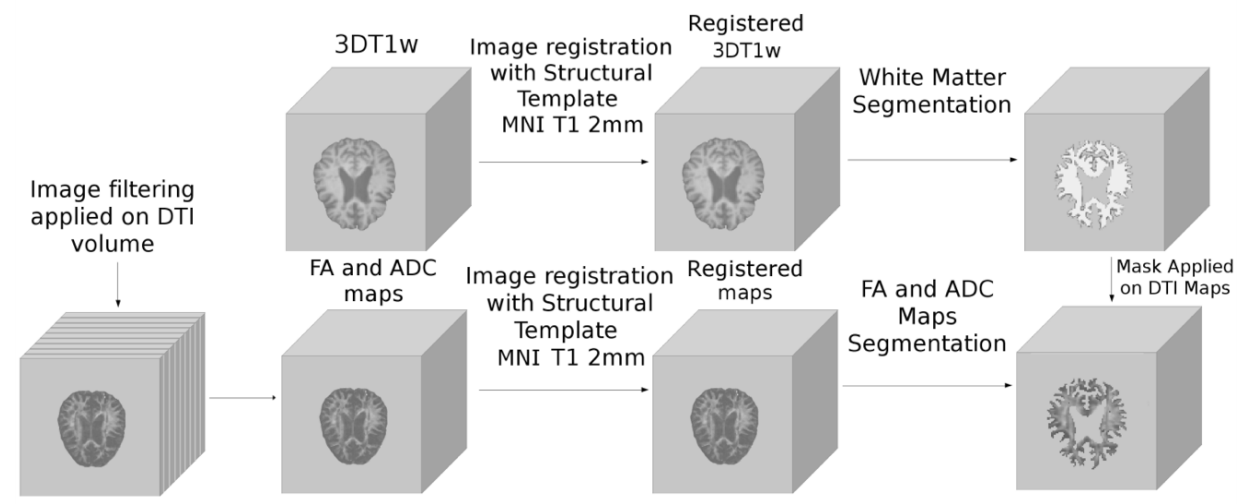

Figure 1. Diagram showing the general image processing applied for white matter segmentation on FA and ADC maps. All the steps were done using FSL software and the spatial filters were only applied on the averaged DTI volumes $(\mathrm{N}=1,2,4,6,8)$. 
improvement, the relative mean error (RME) measure was applied. It worth noting that the RME evaluation could be denoted as a complementary metric in relation to the SSIM results, offering an overall voxel-wise error measure. Both metrics were estimated in FA and ADC maps, considering the segmented WM and all quantitative evaluations were made only on non-zero image voxels, i.e., the image background values were disregarded.

Here, we used the RME formulation based on image voxel-wise space scale calculation, which is usual for several image processing applications (Gonzalez and Woods, 2008). The Equation 4 defines RME used here, where $\mathrm{I}(\mathrm{r})$ is the input image, $\mathrm{R}(\mathrm{r})$ is the reference image, $r(x, y)$ is the voxel coordinates and $(M, N)$ is the size of the image. The reference image for SSIM analysis was the DTI volume with $\mathrm{N}=16$ and for $\mathrm{RME}$ was the $\mathrm{N}=1$. The reason why we chose different $\mathrm{N}$ for each metric is due to the application meaning, which for RME we want to estimate the filters efficiency on a clinical image quality (usually acquired with $\mathrm{N}=1$ ) and for the SSIM we want to compare with the best image quality provided. Additionally, the SSIM improvement could be used for methods that are histogram dependent such as segmentation (de Boer et al., 2009) and also denotes the geometrical preservation presented by the image filtering approach (Wang et al., 2004).

$R M E=\frac{\sum_{x, y}^{M, N}[R(r(x, y))-I(r(x, y))]^{2}}{\sum_{x, y}^{M, N} R(r(x, y))^{2}}$

After the time and conductance parameters definition, the AAD filter needs to define the q-value, which accepts a broad continuous values interval $(0<q<2, q \in \mathfrak{R})$ (Senra et al., 2015). An optimization procedure is required to find the maximum q-value performance, which defines the appropriate diffusion regime. In this step, we used both RME and SSIM as the objective functions to be optimized considering all DWI raw data. The q range was obtained using fixed numerical steps of 0.025 . Five histograms were constructed for the optimum values of each metrics considering images with different numbers of signal averages $(\mathrm{N}=1,2,4,6,8)$. The relative frequency of these histograms indicates the fraction of subjects with minimum/maximum metric value in one specific q-value, e.g., RME/SSIM histogram with 50\% at $\mathrm{q}=0.3$ means that half of the subject's filtered images had the minimum RME and maximum SSIM at $\mathrm{q}=0.3$.

A tractography reconstruction was also performed in order to study the filtering effect over the main tracts pathway. The tractography parameters were: deterministic FA guided pathway estimative, minimum/maximum path length of $20 / 800 \mathrm{~mm}$, stop criteria of 0.25 , and limited angle of $30^{\circ}$. A full-reference metrics were compared with the tractography image to infer the general image distortion reached by each filtering method in relation to the reference result (the unfiltered DTI image). Here we present a quantitative estimative description of the tractography analysis by the coefficient of variation (CV) of the FA value present in six representatives tracts in WM, such as the genu of the corpus callosum (GCC), splenium of the corpus callosum (SCC), corticospinal tract (CST), superior longitudinal fasciculus (SLF), uncinate fasciculus (UNC) and the inferior fronto-occipital fasciculus (IFO) (Wakana et al., 2007). The tractography reconstructions were made in 3D Slicer software (Pieper et al., 2004; Xia et al., 2008), which provides a better control set for image visualization than the FSL tractography toolkit.

Finally, it was also analyzed the FA variability given by the TSA approach (Zhang et al., 2010). The TSA pipeline calculates the statistical differences between groups, where it was conducted using the $\mathrm{N}=16$ DTI volumes as the reference dataset and the same white matter regions given in the previous tractography evaluation. Moreover, using the same tracts region of interest (ROI) as previously described, the effect of each image filtering approach over the tracts count estimate was also studied. The number of fibers could bring more insights about the effects of each filter on post processing techniques that rely on deterministic tractography approach, e.g. whole brain structural connectivity.

\section{Statistical analysis}

Two-tailed t-tests, following data normality test verification with the Kolmogorov-Smirnov, were applied in all image quality evaluation indexes, i.e. RME and SSIM. The tractography representation using $\mathrm{CV}$ analysis used two-tailed t-test on each ROI, in order to infer the differences between raw and filtered data. The TSA analysis performs a t-test group analysis internally, which it was chosen a $p$-value of $p=0.05$ and 5.000 permutations (Zhang et al., 2010).

\section{Results}

\section{q-optimization}

Figure 2 illustrates the histograms obtained with the RME and SSIM indexes. The two well-defined peaks indicate the optimum q values for DTI filtering, where the AAD filter tends to have a balance between optimal values of $\mathrm{q}=0.4$ and $\mathrm{q}=1.4$. However, as we increase the number of averages, the quality metrics become more defined at $\mathrm{q}=0.4$. Therefore, we adopted $\mathrm{q}=0.4$ as the optimum $\mathrm{q}$ value for AAD filter applied to the DTI images. 

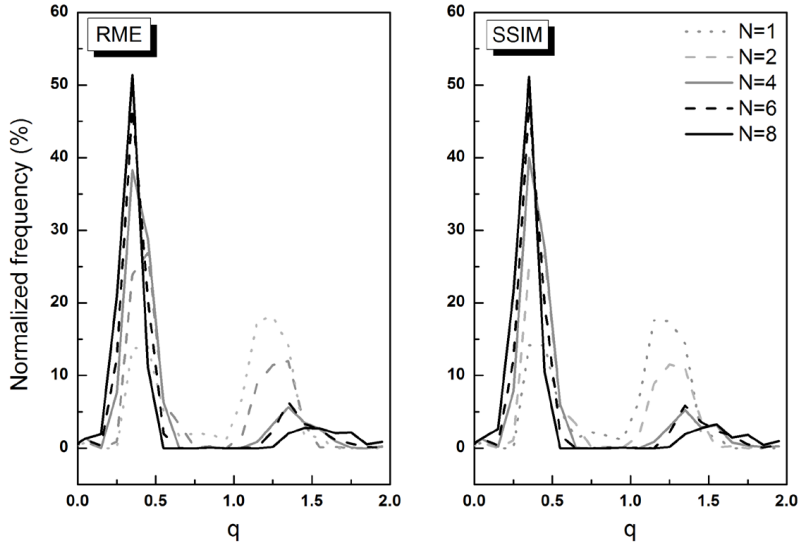

Figure 2. Histograms for optimum q values that minimize the relative mean error (RME) and maximize the structural similarity index (SSIM). The $\mathrm{N}$ values are calculated based on the sequential acquisition order, i.e. the $\mathrm{N}=1$ is the first DTI volume, $\mathrm{N}=2$ is the average of the first two DTI acquisitions, and so on. Maximum frequency in these curves is used to select the optimum q parameter for the AAD filter.

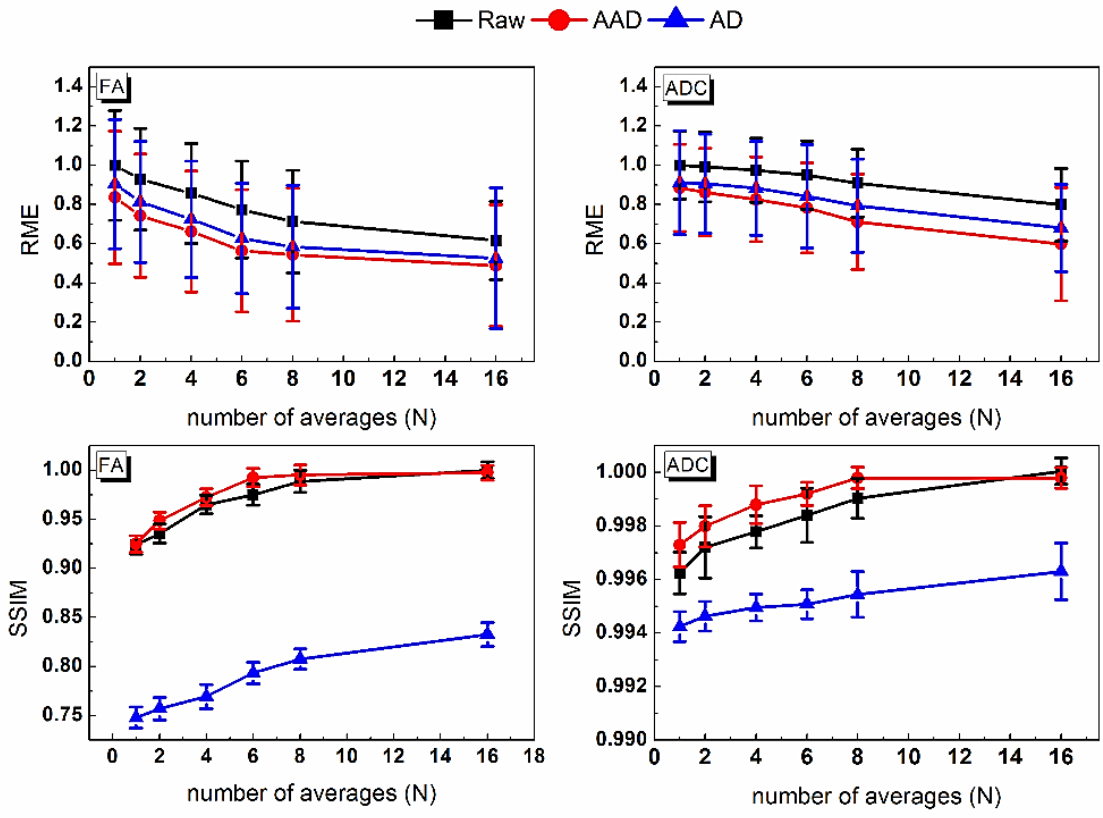

Figure 3. Relative RME and SSIM improvements comparing the anisotropic anomalous diffusion (AAD) filter and the classical anisotropic diffusion (AD) filter. RME improvement used the $\mathrm{N}=1$ image as the reference image, which is the common $\mathrm{N}$ value in clinical routine, and SSIM used the $\mathrm{N}=16$ image due to its high-quality structural definition. The $\mathrm{N}$ calculation follows the same criteria as explained in Figure 2.

\section{Image quality evaluations}

From the FA and ADC maps, both RME improvement and SSIM index for each image volume of $\mathrm{N}$ acquisitions were calculated. The improvement, for RME metric, was estimated as the ratio between the filtered image and the original image with $\mathrm{N}=1$, i.e. without any filter application and with the highest noise component. This analysis was adopted to estimate the overall image quality improvement, regarding the quantitative error, which could be reached with a usual DTI acquisition adopted on clinical routine. In the case of the SSIM, it was adopted the $\mathrm{N}=16$ averages image as the reference image, which helps to compare the image details distortions obtained from each computational filter (using the higher image quality data as the reference image). These operations were applied separately for each $\mathrm{N}$ averaging $(\mathrm{N}=1,2,4,6,8$ and 16) and Figure 3 shows the results for both RME and SSIM. 
The results obtained with the AAD filter showed a consistent improvement for both quantitative maps, FA and ADC. Particularly, the FA maps showed a significant $(\mathrm{p}<0.05)$ higher image quality when compared to the $\mathrm{N}$ averaging approach and $\mathrm{AD}$ filter. These image quality improvements were also obtained for ADC maps, however, with lower differences between each method. As seen in Figure 3, regarding the results obtained with the AAD filter, the SSIM values are close to optimum value, i.e. $\mathrm{SSIM}=1$. Furthermore, $\mathrm{SSIM}$ values are significantly higher for AAD filter when compared to the AD filter $(\mathrm{p}<0.001)$. The SSIM metric is sensible to the tissues borders and geometrical shapes presented in the entire image, which gives a broader difference in FA maps than in ADC map. Additionally, a comparison was also conducted between the quantitative values of FA and ADC maps in six WM regions in DTI images, with and without the application of spatial filters (Table 1).

It worth commenting that the data fluctuation is partially given from the intrinsic biological variability and also due to a low image filtering efficiency. A lower $\mathrm{CV}$ value is not sufficient to determine whether the filtering approach achieved a reasonable result, but the $\mathrm{CV}$ measurement should be compared with the others image quality assessments in order to infer a meaningful conclusion. Hence, a precise image filtering methodology should decrease the noise level and simultaneously preserve the biological structure. Regarding the CV values, the AAD filter demonstrates a decrease of over $30 \%$ with the
FA values $(p=0.05)$, which, on the other hand, the AD filter shows an increase in the $\mathrm{CV}$ values. This increase in $\mathrm{CV}$ given by the $\mathrm{AD}$ filter could be explained mainly by the strong local blurring, mainly in tissue borders, which provides a local value spreading, leading to a higher $\mathrm{CV}$ estimative. In addition, the use of the AAD filter obtained CV measurements comparable with higher $\mathrm{N}$ averages (Table 1), which infers low image distortions. Interestingly, the anomalous paradigm showed a stable image enhancement being independent on image SNR, illustrating a consistent response over different averaging. In general, the AAD filter showed a robust filtering performance for each image quality metric, i.e. CV, RME, and SSIM.

Additionally, the FA and ADC maps are represented in Figure 4 in order to illustrate the difference obtained by each image filtering approach. In general, the differences are highlighted in regions with less dense fiber tracts, mainly between the frontier of the gray and white matter. The $\mathrm{AD}$ filter demonstrates higher distortions on the tissues interfaces which were not evidenced by the AAD filter. Furthermore, the AAD filter showed a consistent correspondence with the $\mathrm{N}=16$ data profile, mainly in FA map. In the case of ADC maps, all the methods showed a regular response, i.e. the $\mathrm{ADC}$ values do not show an intense variation mainly because this quantitative map presents lower contrast than the FA map. The results given in Figure 3 complements what is evidenced in Figure 4. Additionally, in order to give a

Table 1. Coefficient of variation (CV) obtained from the quantitative FA maps with and without filter applications in six different WM regions.

\begin{tabular}{|c|c|c|c|c|c|c|c|c|c|c|c|}
\hline & \multirow{2}{*}{$\mathbf{N}$} & \multirow{2}{*}{ GCC } & \multirow{2}{*}{ SCC } & \multicolumn{2}{|c|}{ CST } & \multicolumn{2}{|c|}{ SLF } & \multicolumn{2}{|c|}{ UNC } & \multicolumn{2}{|c|}{ IFO } \\
\hline & & & & Right & Left & Right & Left & Right & Left & Right & Left \\
\hline \multirow{6}{*}{ Raw } & 1 & 0.427 & 0.423 & 0.435 & 0.375 & 0.198 & 0.248 & 0.248 & 0.286 & 0.380 & 0.332 \\
\hline & 2 & 0.300 & 0.311 & 0.258 & 0.334 & 0.138 & 0.169 & 0.174 & 0.200 & 0.276 & 0.236 \\
\hline & 4 & 0.214 & 0.219 & 0.178 & 0.231 & 0.098 & 0.118 & 0.123 & 0.141 & 0.194 & 0.164 \\
\hline & 6 & 0.175 & 0.180 & 0.145 & 0.188 & 0.081 & 0.096 & 0.100 & 0.115 & 0.159 & 0.135 \\
\hline & 8 & 0.151 & 0.156 & 0.126 & 0.163 & 0.070 & 0.083 & 0.087 & 0.100 & 0.137 & 0.117 \\
\hline & 16 & 0.107 & 0.111 & 0.088 & 0.115 & 0.049 & 0.058 & 0.061 & 0.071 & 0.097 & 0.080 \\
\hline \multirow{6}{*}{ AAD } & 1 & 0.323 & 0.356 & 0.372 & 0.281 & 0.089 & 0.189 & 0.151 & 0.189 & 0.276 & 0.224 \\
\hline & 2 & 0.201 & 0.212 & 0.156 & 0.230 & 0.063 & 0.089 & 0.097 & 0.103 & 0.172 & 0.125 \\
\hline & 4 & 0.113 & 0.120 & 0.106 & 0.128 & 0.059 & 0.061 & 0.065 & 0.073 & 0.089 & 0.078 \\
\hline & 6 & 0.115 & 0.098 & 0.074 & 0.098 & 0.048 & 0.056 & 0.050 & 0.067 & 0.071 & 0.069 \\
\hline & 8 & 0.051 & 0.075 & 0.062 & 0.086 & 0.036 & 0.048 & 0.048 & 0.052 & 0.083 & 0.053 \\
\hline & 16 & 0.058 & 0.051 & 0.049 & 0.051 & 0.024 & 0.025 & 0.036 & 0.036 & 0.065 & 0.042 \\
\hline \multirow{6}{*}{ AD } & 1 & 0.525 & 0.548 & 0.436 & 0.575 & 0.297 & 0.343 & 0.362 & 0.399 & 0.465 & 0.436 \\
\hline & 2 & 0.371 & 0.389 & 0.323 & 0.382 & 0.207 & 0.246 & 0.266 & 0.290 & 0.320 & 0.300 \\
\hline & 4 & 0.275 & 0.286 & 0.245 & 0.296 & 0.167 & 0.186 & 0.201 & 0.218 & 0.233 & 0.234 \\
\hline & 6 & 0.215 & 0.224 & 0.183 & 0.225 & 0.189 & 0.136 & 0.149 & 0.161 & 0.190 & 0.166 \\
\hline & 8 & 0.181 & 0.191 & 0.154 & 0.191 & 0.098 & 0.112 & 0.123 & 0.135 & 0.159 & 0.147 \\
\hline & 16 & 0.137 & 0.141 & 0.118 & 0.145 & 0.079 & 0.088 & 0.091 & 0.101 & 0.127 & 0.118 \\
\hline
\end{tabular}

The values are expressed by the mean of each group, and the AAD and AD filters are applied on all the range of N (1,2, 4, 6, 8 and 16). The highlighted rows illustrate the image quality improvement obtained with AAD in comparison with the raw DTI data. Coefficient of variation (CV) obtained from the quantitative FA maps with and without filter applications in six different WM regions. 

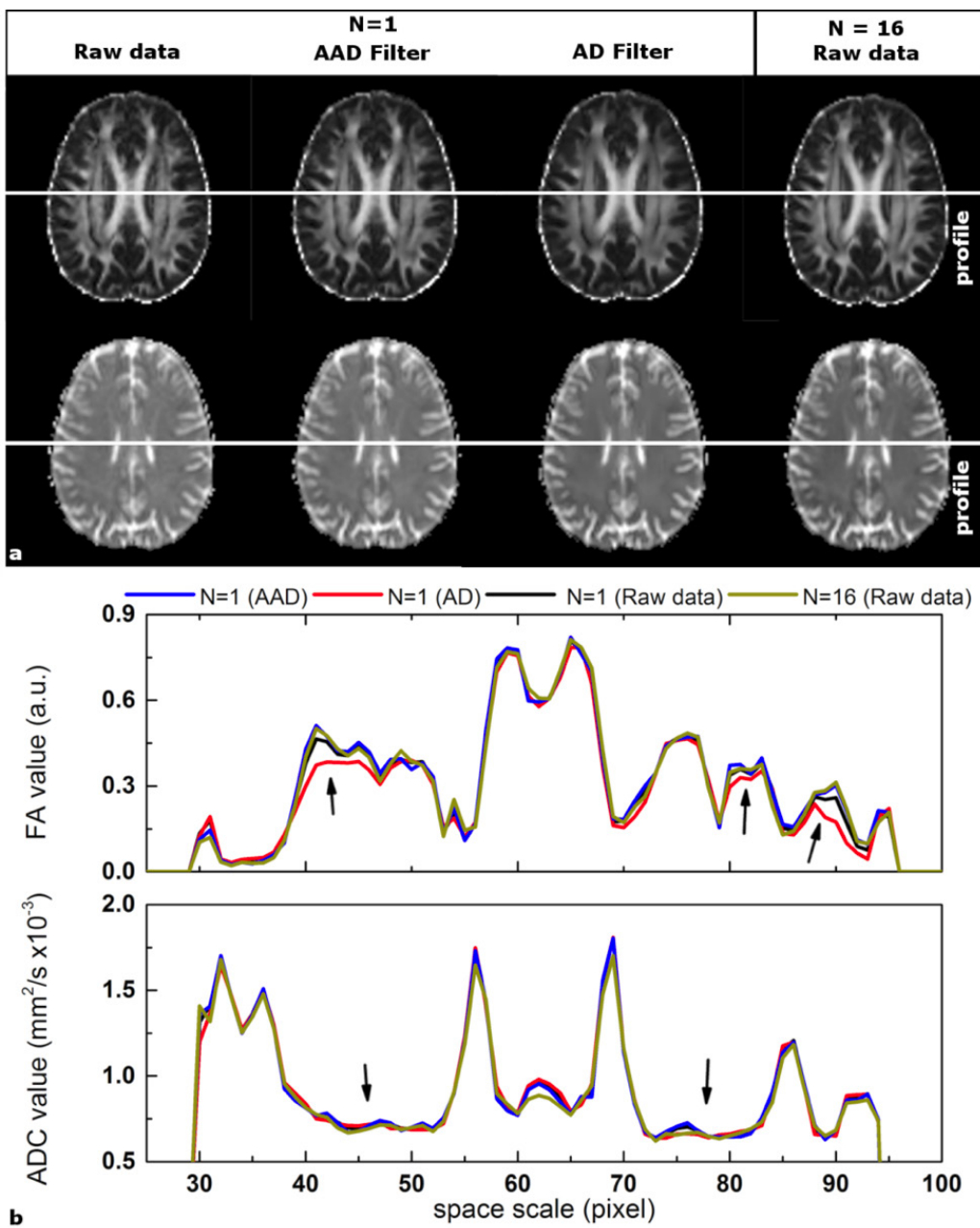

Figure 4. (a) Examples of FA and ADC maps obtained from raw and filtered DTI data with $A A D$ and $A D$ approaches. The raw DTI (N=1), filtered DTI with the AAD $(q=0.4)$ method and the filtered DTI with the classic AD method are compared with the FA map resulting from the reference volume ( $\mathrm{N}=16$ ); (b) A profile is represented to illustrate the FA and ADC values along a medial portion (white lines). Edges preservation are evident in both $\mathrm{AD}$ and $\mathrm{AAD}$ approaches; however, only the $\mathrm{AAD}$ filter is also able to maintain the edge definition in brain regions where there are well-defined transitions between gray and white matter (arrows).

more precise visual comparison between $\mathrm{AD}$ and $\mathrm{AAD}$ results, Figure 5 can be analyzed. As noticed, the general visual improvement achieved by the anomalous paradigm showed a subtle but considerable image enhancement in comparison with the raw data $(\mathrm{N}=1)$. However, more importantly, the $\mathrm{AD}$ general distortions are easier to identify in Figure 5, which complements the quantitative evaluation previously provided. The general tissue anatomical properties seem to be more preserved using AAD filtering method, showing lower noise disturbances and better global brain WM structures maintenance.

In order to compare $\mathrm{AAD}$ and $\mathrm{AD}$ filtering regarding the assessment of fiber representation, two tractography studies were adopted. The TSA measure uses the mean FA value from the $\mathrm{N}=16$ dataset as the baseline values, and the color map illustrated in Figure 6 is regarding the
FA contrast between the $\mathrm{N}=16$ data and the other filtering approaches ( $\mathrm{N}$ averages or computational filtering). In addition, using the same TSA tracts ROIs, white matter deterministic tractographies were reconstructed in order to infer the fiber counting differences between the computational filters and averaging approaches. The fiber counting evaluation is given in Table 2 .

\section{Discussion}

In general, the AAD filter showed a consistent image enhancement, for both FA and ADC maps, which could be highlighted the performance with $\mathrm{N}=6$ and $\mathrm{N}=8$ acquisitions as seen in Figures 2 and 3. This higher filtering performance is reasonable due to less noise on the image data. The Laplacian function is more robust with 

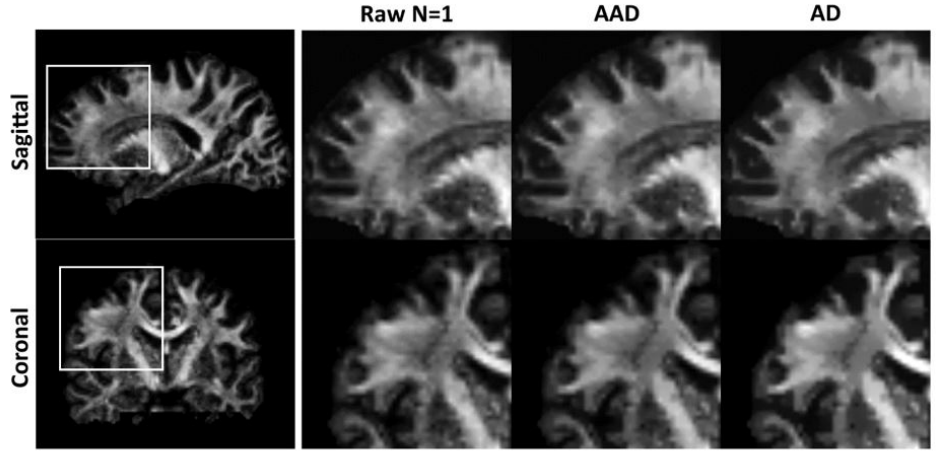

(a)
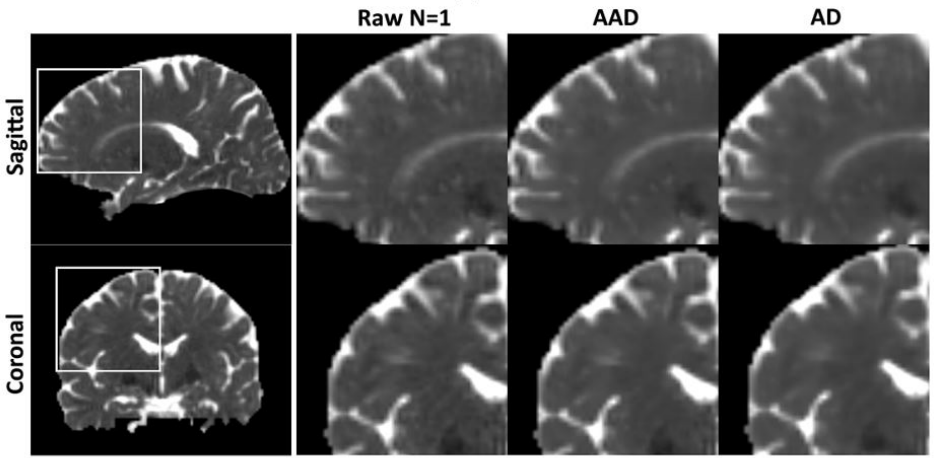

(b)

Figure 5. (a) Illustration of each filtering application using N=1 DTI data. The sagittal and coronal orientations are presented, where amplification is provided in a defined region (white square box). Firstly, as both filters were applied in the axial plane (2D filtering approach), it is clear to see that there is no apparent distortion on the transversal planes. Secondly, it is easier to identify the main filtering artifacts caused by the AD method, showing a stronger blurring on large areas in the white matter tissue, e.g. cortico-spinal tract. The AAD method presents more reliable denoising results, maintaining the general geometrical properties and less noise interference in FA maps. A similar response is found in ADC maps (b).

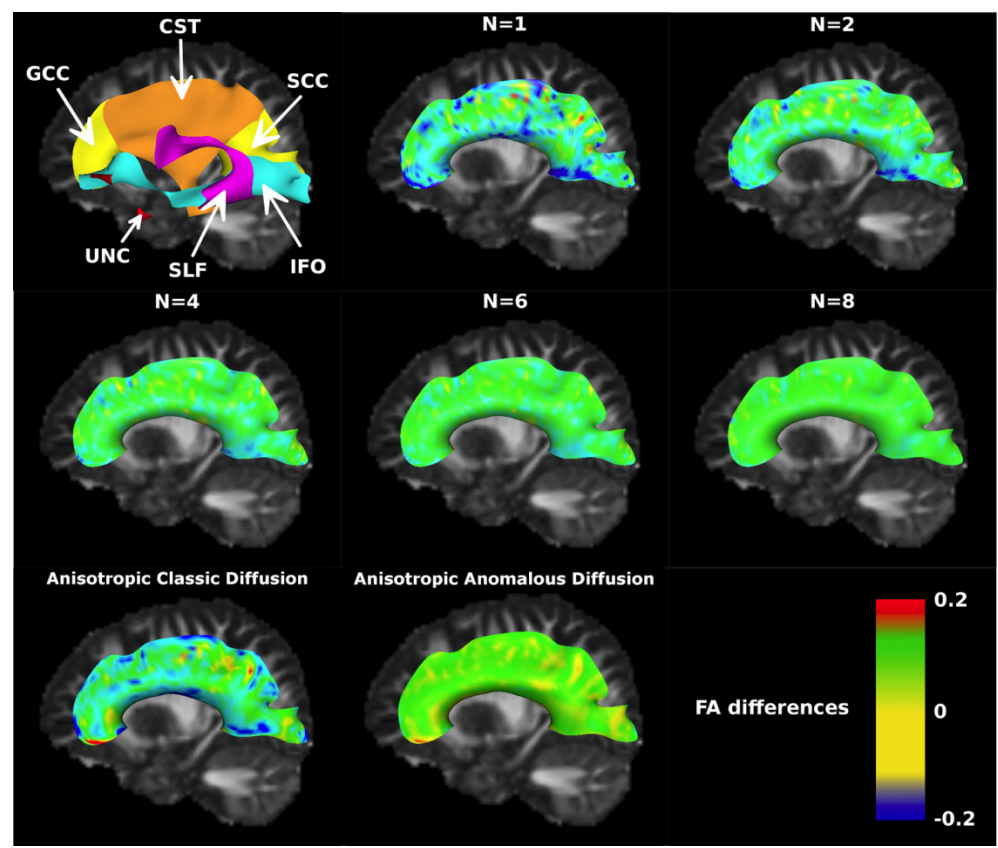

Figure 6. Tract-Specific Analysis (TSA) applied on sequential averages DTI data ( $\mathrm{N}=1,2,4,6$ and 8), AAD and AD filters (both applied over $\mathrm{N}=1 \mathrm{DTI}$ ). The first image (upper left) is the ROIs used in this TSA study, where the following images only represent the corpus callosum region. The AAD filter showed an FA map quality similar to higher $\mathrm{N}$ averages, giving more precise noise attenuation than what is seen in $\mathrm{AD}$ filter. 
Table 2. White matter fiber counting estimates from the deterministic tractography reconstruction in six different WM regions.

\begin{tabular}{ccccccc}
\hline & GCC & SCC & CST & SLF & UNC & IFO \\
\hline $\mathrm{N}=1$ & $62 \pm 28$ & $101 \pm 22$ & $125 \pm 24$ & $30 \pm 5$ & $35 \pm 9$ & $32 \pm 7$ \\
$\mathrm{~N}=2$ & $\mathbf{7 0} \pm \mathbf{2 2}$ & $\mathbf{1 2 3} \pm \mathbf{1 9}$ & $\mathbf{1 4 1} \pm \mathbf{1 9}$ & $\mathbf{3 5} \pm \mathbf{6}$ & $\mathbf{3 3} \pm \mathbf{8}$ & $\mathbf{3 6} \pm \mathbf{8}$ \\
$\mathrm{N}=4$ & $92 \pm 18$ & $141 \pm 15$ & $153 \pm 12$ & $36 \pm 4$ & $34 \pm 6$ & $39 \pm 6$ \\
$\mathrm{~N}=6$ & $105 \pm 12$ & $150 \pm 12$ & $163 \pm 13$ & $35 \pm 4$ & $34 \pm 5$ & $43 \pm 5$ \\
$\mathrm{~N}=8$ & $103 \pm 13$ & $149 \pm 11$ & $165 \pm 12$ & $37 \pm 5$ & $34 \pm 6$ & $44 \pm 5$ \\
$\mathrm{~N}=16$ & $107 \pm 12$ & $151 \pm 12$ & $164 \pm 11$ & $36 \pm 4$ & $34 \pm 6$ & $42 \pm 4$ \\
$\mathrm{AAD}$ & $\mathbf{7 5} \pm \mathbf{2 8}$ & $\mathbf{1 3 2} \pm \mathbf{7}$ & $\mathbf{1 5 8} \pm \mathbf{1 2}$ & $\mathbf{3 6} \pm \mathbf{5}$ & $\mathbf{3 5} \pm \mathbf{1 6}$ & $\mathbf{4 1} \pm \mathbf{3}$ \\
$\mathrm{AD}$ & $65 \pm 35$ & $127 \pm 12$ & $141 \pm 10$ & $26 \pm 7$ & $30 \pm 13$ & $30 \pm 5$
\end{tabular}

The values are expressed by the mean and standard deviation of each group. Units are arbitrary blue and both AAD and AD filters are applied only on $\mathrm{N}=1$ DTI data.

better image quality, leading to better tissue boundaries estimate. However, after the boundaries have been set, the local spatial noise filtering is assumed regarding the classical Gaussian (AD filter) or q-Gaussian (AAD filter) paradigms.

It is worth mentioning that the AAD filter shows consistently better results in comparison with the $\mathrm{AD}$ filter, indicating a better filtering modulation given by the anomalous diffusion paradigm (Senra et al., 2013, 2015). Moreover, the AAD filter response over low $\mathrm{N}$ averaging also shows an interesting result, in which the overall image quality could be enhanced to a quality level equivalent to an image with higher numbers of averages. Figure 3 shows the comparative improvements and absolute index value (for RME and SSIM, respectively) obtained with and without each filtering approach, according to $\mathrm{N}$. We noticed that $\mathrm{AAD}$ filter provides an image quality statistically equivalent $(\mathrm{p}<0.05)$ to images obtained with higher N. In general, a decrease in RME was generally obtained as an equivalent of two additional acquisitions, i.e. a $\mathrm{N}=1$ image that was filtered by AAD filter has an equivalent image quality that would be achieved by a $\mathrm{N}=3$ averages.

In our previous study, it was observed that $\mathrm{q}$ $>1$ is more suitable to filter structural MRI images (Senra et al., 2015). However, the noise intensity in DTI led us to adopt $\mathrm{q}<1$, as seen in Figure 2. The q-Gaussian defined with $\mathrm{q}<1$ offers a robust neighborhood voxel intensity regulation with restrict local influence, given by the compact support probability density function. For this reason, the restrict diffusion offers a partial solution to high noise intensity level presented in DTI data. Specifically, we found $\mathrm{q}=0.4$ as optimum q value for DTI processing, and thus subdiffusion is more advantageous for DTI images.

The high influence of imaging artifacts such as partial volume effect, magnetic pulse inhomogeneity, and noise level, naturally present in DTI images, can offer barriers to both anomalous and classical spatial filters. However, even with these confounding components, the AAD filter was more robust than the AD approach. The same edge function (Equation 2) for both image filtering methods was applied in this study, showing that the main difference resulted from each image filter approach is basically obtained from the voxel intensity probability distribution purposed by each filter. In other words, the q-Gaussian distribution with compact support, given by the AAD method, showed a better noise attenuation, seen in the FA and ADC measures. On the contrary, the Gaussian smoothing, given by the AD method, apply a higher weighting over far voxels, which is characteristic for long tail probability distribution function. Hence, the anomalous filtering method results in better edge preservation and less quantitative distortions, mainly due to the q-Gaussian short tail properties.

In summary, the AAD filter is robust and consistent with both FA and ADC maps. Several brain regions present strong distortions when the AD filter is applied, such as the corpus callosum and the internal capsule. Furthermore, the $\mathrm{AD}$ filter presented poor control over tissue transition mainly in the interface between white and gray matter, as seen in Figure 4, 5 and 6. The central region on each ROI appears strongly smoothed with the $\mathrm{AD}$ filter, which it is considered as a homogenous region by the classical filtering paradigm. In fact, some image artifact may influence the general filtering performance, e.g. magnetic field inhomogeneity and head motion, which smoothly distorts a low level of voxel intensity throughout the image space. A spatial filtering approach should provide a controlled handling of this low-level interference, being capable of reducing the noise level without removing fine details of the image. As noticed, the AAD method was more promising than $\mathrm{AD}$ approach, as seen in Figure 5 and 6. Furthermore, it is highly probable to find crossing-fibers regions in the brain WM, being naturally presented as an inhomogeneous feature in the DTI maps (Tuch et al., 2003). An intense smoothing with relative edge preservation does not imply preservation of the local biological environment, which may cause serious interference on further DTI analysis 
such as structural brain connectivity (Van den Heuvel and Hulshoff Pol, 2010).

On the other hand, such an intense filtering artifact is not significantly presented in the AAD filter. The AAD filter showed as a suitable method to maintain the edge definition in brain regions where exist well-defined transitions between gray and white matter tissues. As noticed in Figure 4, the AAD filter provides an FA profile that has a close similarity to the $\mathrm{N}=16 \mathrm{FA}$ results. The difference in FA and ADC maps reconstruction is related to the filtering effect over the eigenvalues and eigenvector estimation from DTI reconstruction. Remember that all filtering methods were only applied to the raw DTI data, providing insights about the filtering response on the diffusion gradients volumes. In general, the classical filtering approach distorts the tensorial information, resulting in a lower FA estimative. However, these image distortions do not offer a higher impact on the ADC maps mainly because it uses the mean eigenvalues to reconstruct this type of image.

The TSA estimative add another advantage to the image enhancement achieved by the AAD filter, in comparison for $\mathrm{N}=1$ and $\mathrm{AD}$ filter. Firstly, it could be noticed that the higher contrasts are seen in raw $\mathrm{N}=1$ data, which is expected by the intense noise component. As the number of averages rises, the variability of the FA values decreases. Secondly, the results obtained by the AAD filter showed a consistent FA enhancement for the corpus callosum tracts and, for the other white matter tracts, the response was similar.

In general, the tractography evaluation also confirms the distortions showed by the AD filter, as shown in Table 2 . As noticed, the major white matter tracts resulting from AAD filter reveal a favorable image filtering application for the anomalous paradigm. For instance, taking the mean fiber counting values at GCC and SCC regions, the $\mathrm{N}=1$ acquisition showed 62 fibers and 101 fibers; $\mathrm{N}=16$ acquisitions showed 107 and 151 fibers; AAD filtered showed 75 and 132 fibers and AD filtered showed 65 and 127 fibers. The same pattern repeats for the other white matter tracts. In fact, the major difference presented in Table 2 appear between the AD filter and the other image filtering approaches, i.e. the $\mathrm{N}=16$ averages and the AAD filter. In general, the AD filter shows lower fiber counting than the AAD filter, which the AAD filter tends to give a slight advantage in comparison with the raw $\mathrm{N}=1$ tractography, offering less variability and a total fiber counting comparable with higher $\mathrm{N}$ averages.

In conclusion, investments in scanner hardware for DTI image improvement are, in many cases, economically impractical and for this reason, a commonly adopted strategy is the acquisition of multiple images in DTI exams. However, there is often a practical limitation related with the increased acquisition time. Recently, several studies using computational tools for noise attenuation were applied in order to improve the quality and accuracy of DTI scans. However, the widely used classic anisotropic filtering paradigm does not offer promising results for noisy images, especially for DTI images. In this study, we show a significant quality improvement in visual and quantitative measurements when the AAD filter is applied. In general, it is observed that DTI images processed with the AAD filter with $\mathrm{q}=0.4$ present a final image quality similar to what is seen in DTI images with more acquisition averaging. Finally, the AAD filter is a promising method that could help other applications in medical diagnosis and neuroscience research.

\section{Acknowledgements}

The authors would like to thank CAPES (Coordenação de Aperfeiçoamento de Pessoal de Nível Superior) and $\mathrm{CNPq}$ (Conselho Nacional de Desenvolvimento Científico e Tecnológico - 201871/2015-7/SWE) - Brazilian financial agencies, for the grants provided.

\section{References}

Aja-Fernandez S, Niethammer M, Kubicki M, Shenton ME, Westin C-F. Restoration of DWI data using a Rician LMMSE estimator. IEEE Transactions on Medical Imaging. 2008; 27(10):1389-403. PMid:18815091. http://dx.doi.org/10.1109/ TMI.2008.920609.

Alexander AL, Lee JE, Lazar M, Field AS. Diffusion tensor imaging of the brain. Neurotherapeutics; the Journal of the American Society for Experimental NeuroTherapeutics. 2007; 4(3):316-29. PMid:17599699. http://dx.doi.org/10.1016/j. nurt.2007.05.011.

Bai J, Feng X-C. Fractional-order anisotropic diffusion for image denoising. IEEE Transactions on Image Processing. 2007; 16(10):2492-502. PMid:17926931. http://dx.doi.org/10.1109/ TIP.2007.904971.

Basu S, Fletcher T, Whitaker R. Rician noise removal in diffusion tensor MRI. Lecture Notes in Computer Science. 2006; 4190:117-25. http://dx.doi.org/10.1007/11866565_15.

Boer R, Vrooman HA, van der Lijn F, Vernooij MW, Ikram MA, van der Lugt A, Breteler MM, Niessen WJ. White matter lesion extension to automatic brain tissue segmentation on MRI. NeuroImage. 2009; 45(4):1151-61. PMid:19344687. http://dx.doi.org/10.1016/j.neuroimage.2009.01.011.

Bullmore E, Barnes A, Bassett DS, Fornito A, Kitzbichler M, Meunier D, Suckling J. Generic aspects of complexity in brain imaging data and other biological systems. NeuroImage. 2009; 47(3):1125-34. PMid:19460447. http://dx.doi.org/10.1016/j. neuroimage.2009.05.032.

Chen B, Hsu EW. Noise removal in magnetic resonance diffusion tensor imaging. Magnetic Resonance in Medicine. 2005; 54(2):393-401. PMid:16032670. http://dx.doi.org/10.1002/ mrm. 20582 . 
Christiansen O, Lee T-M, Lie J, Sinha U, Chan TF. Total variation regularization of matrix-valued images. International Journal of Biomedical Imaging. 2007; 2007:1. PMid:18256729. http://dx.doi.org/10.1155/2007/27432.

Gonzalez RC, Woods RE. Digital Image Processing. New Jersey: Prentice Hall; 2008. v. 14.

Jenkinson M, Beckmann CF, Behrens TEJ, Woolrich MW, Smith SM. FSL. NeuroImage. 2012; 62(2):782-90. PMid:21979382. http://dx.doi.org/10.1016/j.neuroimage.2011.09.015.

Kiselev VG, Hahn KR, Auer DP. Is the brain cortex a fractal? NeuroImage. 2003; 20(3):1765-74. PMid:14642486. http:// dx.doi.org/10.1016/S1053-8119(03)00380-X.

Lee JE, Chung MK, Alexander AL. Evaluation of anisotropic filters for diffusion tensor imaging. In: Proceedings of the $3 \mathrm{rd}$ IEEE International Symposium on Biomedical Imaging: Nano to Macro; 2006; Arlington, VA, USA. USA: IEEE; 2006. p. 77-80. v. $1-3$.

Lin W, Jay Kuo C-C. Perceptual visual quality metrics: a survey. Journal of Visual Communication and Image Representation. 2011;22(4):297-312. http://dx.doi.org/10.1016/j.jvcir.2011.01.005.

Ling J, Bovik AC. Smoothing low-SNR molecular images via anisotropic median-diffusion. IEEE Transactions on Medical Imaging. 2002; 21(4):377-84. PMid:12022625. http://dx.doi. org/10.1109/TMI.2002.1000261.

Malacarne L, Mendes R, Pedron I, Lenzi E. Nonlinear equation for anomalous diffusion: unified power-law and stretched exponential exact solution. Physical Review E: Statistical, Nonlinear, and Soft Matter Physics. 2001; 63(3 Pt 1):30101. PMid:11308617. http://dx.doi.org/10.1103/PhysRevE.63.030101.

Manjón JV, Carbonell-Caballero J, Lull JJ, García-Martí G, Martí-Bonmatí L, Robles M. MRI denoising using NonLocal Means. Medical Image Analysis. 2008; 12(4):514-23. PMid:18381247. http://dx.doi.org/10.1016/j.media.2008.02.004.

Martin-Fernandez M, Munoz-Moreno E, Cammoun L, Thiran JP, Westin CF, Alberola-Lopez C. Sequential anisotropic multichannel Wiener filtering with Rician bias correction applied to 3D regularization of DWI data. Medical Image Analysis. 2009; 13(1):19-35. PMid:18639481. http://dx.doi. org/10.1016/j.media.2008.05.004.

Maximov II, Farrher E, Grinberg F, , Shah NJ. Spatially variable Rician noise in magnetic resonance imaging. Medical Image Analysis. 2012; 16(2):536-48. PMid:22209560. http://dx.doi. org/10.1016/j.media.2011.12.002.

McGraw T, Vemuri BC, Chen Y, Rao M, Mareci T. DT-MRI denoising and neuronal fiber tracking. Medical Image Analysis. 2004; 8(2):95-111. PMid:15063860. http://dx.doi.org/10.1016/j. media.2003.12.001

Moraschi M, Hagberg GE, Di Paola M, Spalletta G, Maraviglia B, Giove F. Smoothing that does not blur: effects of the anisotropic approach for evaluating diffusion tensor imaging data in the clinic. Journal of Magnetic Resonance Imaging. 2010; 31(3):690-7. PMid:20187214. http://dx.doi.org/10.1002/ jmri.22040.

Perona P, Malik J. Scale-space and edge detection using anisotropic diffusion. IEEE Transactions on Pattern Analysis and Machine Intelligence. 1990; 12(7):629-39. http://dx.doi. org/10.1109/34.56205.

Pieper S, Halle M, Kikinis R. 3D slicer. In: Proceedings of the 1st IEEE International Symposium on Biomedical Imaging: From Nano to Macro; 2004; Arlington, Virginia, USA. USA: IEEE; 2004.

Schwämmle V, Nobre FD, Tsallis C. q-Gaussians in the porous-medium equation: stability and time evolution. The European Physical Journal B. 2008; 66(4):537-46. http:// dx.doi.org/10.1140/epjb/e2008-00451-y.

Senra ACS Fo. Anomalous Filters module to 3D Slicer software [internet]. 3D Slicer Wiki; 2017. [cited 2017 Aug 21]. Available from: http://www.slicer.org/wiki/Documentation/Nightly/ Extensions/AnomalousFilters

Senra AC Fo, Barbosa JHO, Salmon CEGS, Murta LO. Anisotropic anomalous diffusion filtering applied to relaxation time estimation in magnetic resonance imaging. In: Proceedings of the Annual International Conference of the IEEE Engineering in Medicine and Biology Society; 2014; Chicago, IL, USA. USA: IEEE; 2014. p. 3893-6.

Senra AC Fo, Duque JJ, Murta LO. Isotropic anomalous filtering in Diffusion-Weighted Magnetic Resonance Imaging. In: Proceedings of the Conference of the IEEE Engineering in Medicine and Biology Society. 2013; Osaka. USA: IEEE; 2013. p. 4022-5.

Senra AC Fo, Garrido Salmon CE, Murta LO Jr. Anomalous diffusion process applied to magnetic resonance image enhancement. Physics in Medicine and Biology. 2015; 60(6):2355-73. PMid:25716129. http://dx.doi.org/10.1088/0031$9155 / 60 / 6 / 2355$.

Senra ACS, Murta LO Jr. Anomalous diffusion paradigm for image denoising process [internet]. Insight-Journal; 2017. [cited 2017 Aug 21]. Available from: http://www.insight-journal.org/ browse/publication/980

Shenton ME, Hamoda HM, Schneiderman JS, Bouix S, Pasternak O, Rathi Y, Vu MA, Purohit MP, Helmer K, Koerte I, Lin AP, Westin CF, Kikinis R, Kubicki M, Stern RA, Zafonte R. A review of magnetic resonance imaging and diffusion tensor imaging findings in mild traumatic brain injury. Brain Imaging and Behavior. 2012; 6(2):137-92. PMid:22438191. http://dx.doi.org/10.1007/s11682-012-9156-5.

Tristán-Vega A, Aja-Fernández S. DWI filtering using joint information for DTI and HARDI. Medical Image Analysis. 2010; 14(2):205-18. PMid:20005152. http://dx.doi.org/10.1016/j. media.2009.11.001.

Tsallis C, Lenzi EK. Anomalous diffusion: nonlinear fractional Fokker-Planck equation. Chemical Physics. 2002; 284(12):341-7. http://dx.doi.org/10.1016/S0301-0104(02)00557-8.

Tsallis C. Introduction to nonextensive statistical mechanics: approaching a complex world. New York: Springer; 2009. v. 1.

Tsiotsios C, Petrou M. On the choice of the parameters for anisotropic diffusion in image processing. Pattern Recognition. 2013; 46(5):1369-81. http://dx.doi.org/10.1016/j.patcog.2012.11.012.

Tuch DS, Reese TG, Wiegell MR, Wedeen VJ. Diffusion MRI of complex neural architecture. Neuron. 2003; 40(5):885-95. PMid:14659088. http://dx.doi.org/10.1016/S0896-6273(03)00758-X. 
Van den Heuvel MP, Hulshoff Pol HE. Exploring the brain network: a review on resting-state fMRI functional connectivity. European Neuropsychopharmacology. 2010; 20(8):519-34. PMid:20471808. http://dx.doi.org/10.1016/j. euroneuro.2010.03.008.

Wakana S, Caprihan A, Panzenboeck MM, Fallon JH, Perry M, Gollub RL, Hua K, Zhang J, Jiang H, Dubey P, Blitz A, van Zijl P, Mori S. Reproducibility of quantitative tractography methods applied to cerebral white matter. NeuroImage. 2007; 36(3):630-44. PMid:17481925. http://dx.doi.org/10.1016/j. neuroimage.2007.02.049.

Wang Z, Bovik AC, Sheikh HR, Simoncelli EP. Image quality assessment: from error visibility to structural similarity. IEEE Transactions on Image Processing. 2004; 13(4):600-12. PMid:15376593. http://dx.doi.org/10.1109/TIP.2003.819861.
Xia T, Baird C, Jallo G, Hayes K, Nakajima N, Hata N, Kazanzides P. An integrated system for planning, navigation and robotic assistance for skull base surgery. International Journal of Medical Robotics and Computer Assisted Surgery. 2008; 4(4):321-30. PMid:18803337. http://dx.doi.org/10.1002/rcs.213.

Xu Q, Anderson AW, Gore JC, Ding Z. Efficient anisotropic filtering of diffusion tensor images. Magnetic Resonance Imaging. 2010; 28(2):200-11. PMid:20061113. http://dx.doi. org/10.1016/j.mri.2009.10.001.

Zhang H, Awate SP, Das SR, Woo JH, Melhem ER, Gee JC, Yushkevich PA. A tract-specific framework for white matter morphometry combining macroscopic and microscopic tract features. Medical Image Analysis. 2010; 14(5):666-73. PMid:20547469. http://dx.doi.org/10.1016/j.media.2010.05.002. 